\title{
Hipotermia terapéutica en recién nacidos con encefalopatía hipóxico-isquémica
}

\author{
Therapeutic hypothermia in newborns with ischemic hypoxic encephalopathy
}

\author{
Araceli Martínez-Hernández, ${ }^{*+}$ Rodrigo Barrón-San Pedro, ${ }^{\ddagger}$ Saúl Jesús Garza-Morales, ${ }^{\ddagger}$ \\ Abril Ariadna De la Cruz-Real, ${ }^{\ddagger}$ Mario Enrique Rendón-Macías, ${ }^{\S}$ Mónica Magdalena Hidalgo-Vázquez, \\ Karen Plascencia-Pimentel, ${ }^{\llbracket}$ José Iglesias-Leboreiro, ${ }^{\ddagger}$ Isabel Bernárdez-Zapata ${ }^{\ddagger}$ \\ * Residente de Neonatología, Facultad Mexicana de Medicina, Universidad La Salle México; ${ }^{\ddagger}$ Hospital Español de México; \\ $\S$ Universidad Panamericana, Escuela de Medicina; " Residente de Neonatología, Hospital Español de México, Ciudad de México.
}

\section{RESUMEN}

Introducción: La encefalopatía hipóxico-isquémica (EHI) es un síndrome de disfunción neurológica secundaria a la falta de oxigenación del cerebro alrededor del nacimiento. La hipotermia terapéutica ha reducido la mortalidad neonatal y la discapacidad neurológica. Objetivos: Describir la evolución de recién nacidos (RN) con EHI manejados con hipotermia terapéutica durante 72 horas. Material y métodos: En un periodo de cinco años, $12 \mathrm{RN}>34$ semanas de gestación (SDG) con EHI moderada y grave fueron sometidos a hipotermia terapéutica; ocho con hipotermia corporal total y cuatro a enfriamiento craneal selectivo. Las variables analizadas fueron: mortalidad intrahospitalaria, así como las condiciones neurológicas al egreso hospitalario, tanto clínicas como de estudios de resonancia magnética (IRM) y de estudios de neurofisiología. Resultados: Los $12 \mathrm{RN}$ iniciaron la hipotermia terapéutica antes de las seis horas de vida y la recibieron por 72 horas. Fallecieron dos pacientes después de la hipotermia. Ocho pacientes se egresaron sin datos de alarma o focalización neurológica. La IRM fue normal en cinco, en tres se reportó edema y dos presentaron infartos cerebrales. En cinco pacientes los potenciales auditivos, visuales y somatosensoriales fueron normales; mientras que los cinco restantes tuvieron alteraciones en la vía auditiva. Conclusiones: La hipotermia terapéutica, mediante hipotermia corporal total o enfriamiento craneal, en RN con $\mathrm{EHI}$ moderada y grave parece ser efectiva para limitar el daño neurológico.

Palabras clave: Hipotermia terapéutica, encefalopatía hipóxico-isquémica, recién nacidos.

\begin{abstract}
Introduction: Hypoxic-ischemic encephalopathy (HIE) is a syndrome of neurological dysfunction secondary to lack of oxygenation of the brain around birth. Therapeutic hypothermia has reduced neonatal mortality and neurological disability. Objectives: To describe the clinical course of neonates with HIE treated with therapeutic hypothermia for 72 hours. Material and methods: In a five-year period, twelve neonates > 34 weeks gestational age, with moderate and severe HIE were treated with therapeutic hypothermia; eight with total body hypothermia and four with selective cranial cooling. Variables recorded were: in-hospital mortality, as well as neurological conditions at hospital discharge, both clinical and those obtained from magnetic resonance imaging (MRI) and neurophysiological studies. Results: The twelve neonates started therapeutic hypothermia before six hours of life, maintaining it for 72 hours. Two patients died after hypothermia. Eight patients were discharged without evidence of neurological impairment. MRI was normal in five patients; three had cerebral edema and two patients had cerebral infarcts. Auditory, visual and somatosensory potentials were normal in five patients; while the rest had alterations in the auditory pathway. Conclusions: Therapeutic hypothermia by total body hypothermia or head cooling in infants with moderate and severe EHI appear to be effective in limiting neurological damage.
\end{abstract}

Keywords: Therapeutic hypothermia, hypoxic-ischemic encephalopathy, newborn.

+ Correspondencia: AMH, ara.mar33@gmail.com

Conflicto de intereses: Los autores declaran que no tienen.

Citar como: Martínez-Hernández A, Barrón-San Pedro R, Garza-Morales SJ, De la Cruz-Real AA, Rendón-Macías ME, Hidalgo-Vázquez MM et al. Hipotermia terapéutica en recién nacidos con encefalopatía hipóxico-isquémica. Rev Mex Pediatr. 2020; 87(5): 176-182. https:// dx.doi.org/10.35366/97171 


\section{INTRODUCCIÓN}

La encefalopatía hipóxico-isquémica (EHI) es un síndrome clínico de disfunción neurológica secundaria a falta de oxigenación del cerebro, en el periodo perinatal. Es una de las principales causas de mortalidad y discapacidad neurológica permanente. En el ámbito mundial se estima una incidencia de uno a ocho casos por cada 1,000 recién nacidos $(\mathrm{RN})$ vivos de término, pero en países subdesarrollados la incidencia es mayor. ${ }^{1}$

En Latinoamérica, datos epidemiológicos señalan que en Colombia la prevalencia de asfixia perinatal es de tres casos por cada 1,000 RN vivos, con una mortalidad del $40 \%$ y con discapacidad neurológica del 70\%. ${ }^{2}$ Mientras que en Ecuador, en el año 2016, la asfixia perinatal ocupó el sexto lugar como causa de mortalidad infantil, el número de muertes fue de 112 de un total de 3,042 defunciones de niños menores de un año. ${ }^{3}$ En México, en el 2003 se registraron 2,271,700 nacimientos y 20,806 defunciones neonatales, la principal causa de muerte en el periodo neonatal fue asfixia con 10,277 decesos, lo que representa el $49.4 \%$ del total de las defunciones. ${ }^{4}$

La lesión cerebral por EHI consta de varias fases. La primera es una falla energética primaria con despolarización celular, acumulación de lactato, fosfato inorgánico y muerte neuronal. Después, se presenta una reperfusión con recuperación parcial del metabolismo oxidativo del cerebro (fase latente), tras ésta, el metabolismo puede deteriorarse nuevamente por alteración en la función mitocondrial, dando lugar a muerte neuronal. ${ }^{5}$ Debido al lento desarrollo de la lesión cerebral, las intervenciones terapéuticas en la fase de latencia han mostrado aminorar la lesión cerebral. Una de ellas es la hipotermia terapéutica para $\mathrm{RN}>$ 35 semanas de gestación (SDG).

La hipotermia terapéutica (HT) consiste en reducir la temperatura corporal del neonato de forma controlada $\left(33\right.$ a $\left.34{ }^{\circ} \mathrm{C}\right)$, entre las primeras seis y 72 horas de vida, seguida de recalentamiento lento. Los resultados de diversas investigaciones alrededor del mundo han mostrado que la HT reduce la mortalidad neonatal, así como la discapacidad ocasionada por la EHI grave. ${ }^{6-11}$ En Latinoamérica existe escasa experiencia sobre el uso de HT. En una cohorte colombiana de 64 pacientes publicada en el 2018, esta estrategia permitió la supervivencia del $81 \%$, en quienes se inició antes de las primeras 13 horas de vida. ${ }^{2}$

La HT ha sido implementada bajo dos estrategias: la hipotermia corporal total y el enfriamiento craneal selectivo, ambas parecen conferir beneficio para la protección del daño cerebral. Se conoce que los equipos de enfriamiento con servocontrol de temperatura son los que garantizan temperaturas más estables y menores fluctuaciones, en comparación a los que tienen ajuste manual; además, requieren menor participación del equipo médico y de enfermería. ${ }^{12}$

En México, en el momento de realizar la presente investigación, no se encontraron reportes sobre su uso. El objetivo de este estudio es analizar la supervivencia y condición neurológica al momento del alta de $\mathrm{RN}$ ingresados a una Unidad de Cuidados Intensivos Neonatales (UCIN) con diagnóstico de EHI moderada y grave tratados con HT.

\section{MATERIAL Y MÉTODOS}

Se trata de un estudio retrospectivo de pacientes sometidos al protocolo de HT, bajo dos modalidades: hipotermia corporal total (HCT) y enfriamiento craneal selectivo (ECS), durante el periodo de marzo de 2015 al diciembre del 2019. Se incluyeron los pacientes que cumplieron con los siguientes tres criterios:

1) $\mathrm{RN}>34 \mathrm{SDG}$, con un peso al nacimiento $>1,800 \mathrm{~g}$ y con al menos seis horas de vida.

2) $\mathrm{pH}$ sérico $\leq 7.0$ o un déficit de base de $\geq 16 \mathrm{mmol} / \mathrm{L}$, en sangre umbilical, $\mathrm{pH}$ sérico de 7.01 a 7.15 o un déficit de base entre 10 a $15.9 \mathrm{mmol} / \mathrm{L}$ en sangre venosa durante la primera hora de vida. Cuando no se tenían datos de gasometría, la historia de un evento perinatal adverso grave (ej: prolapso de cordón, desprendimiento de placenta), puntaje de Apgar $\leq 5$ a los 10 minutos o requerimiento de ventilación a los 10 minutos de vida.

3) Evidencia de EHI moderada a grave, de acuerdo con clasificación de Sarnat, o presencia de crisis convulsivas clínicas, con o sin electroencefalograma (EEG) con trazo anormal por más de 20 minutos en la actividad de base, o bien, actividad epiléptica después de la primera hora de vida.

Se excluyeron pacientes con malformaciones congénitas mayores o cromosomopatías incompatibles con la vida, así como $\mathrm{RN}$ con restricción del crecimiento intrauterino $(<1,800 \mathrm{~g})$.

Una vez cumplidos los criterios de selección y posterior al consentimiento informado escrito por los padres o tutores, se procedió a iniciar la HT. La modalidad fue de acuerdo con el periodo; los primeros casos fueron 
manejados con ECS y, a partir del año 2018, fue con HCT, momento en que estuvo disponible en nuestra unidad. En todos los casos la HT tuvo una duración de 72 horas.

Maniobras. La HT se realizó con el objetivo de lograr una temperatura rectal entre $33-34{ }^{\circ} \mathrm{C}$. Para la HCT se utilizó el equipo TECOTHERM NEO ${ }^{\circledR}$, el cual consta de un colchón envolvente por donde fluye agua fría con una velocidad variable, la cual cambia de acuerdo con la temperatura registrada cada segundo mediante una sonda rectal. Por su parte, para el ECS se empleó equipo Cool-Cap ${ }^{\circledR}$, que consiste en un gorro de hipotermia donde fluye el líquido de enfriamiento. La temperatura se vigila con cinco sensores (rectal, piel sobre la región hepática, cuero cabelludo y otras dos localizaciones opcionales). El ajuste del equipo fue manual y bajo vigilancia del equipo de salud.

En ambos modelos, el procedimiento de HT consistió en tres fases: inducción, mantenimiento y recalentamiento. La inducción se inició en la sala de partos o al ingreso a la UCIN con hipotermia pasiva (calentador radiante de la cuna, apagado), mientras se instaló el equipo de enfriamiento. Una vez conectado, se alcanzaba la temperatura diana en alrededor de una hora. El mantenimiento de la hipotermia se realizó durante 72 horas de vida. Al término de este periodo, se inició el calentamiento paulatino con incrementos de 0.2 a $0.5^{\circ} \mathrm{C}$ por hora, hasta llegar a la normotermia, en un plazo mínimo de seis y máximo de 17 horas. Durante la fase de calentamiento, algunos pacientes recibieron sedación y analgesia con morfina a dosis bajas o infusión de fentanilo.

Durante el protocolo de enfriamiento, los pacientes fueron vigilados en la UCIN con registro de electroencefalograma continuo de amplitud controlada (aEEG). Además, diariamente se tomaron los siguientes estudios de laboratorio: biometría hemática, electrolitos séricos, química sanguínea, pruebas de función hepática, tiempos de coagulación, gasometría y lactato. Todos los pacientes recibieron eritropoyetina $1,000 \mathrm{UI} / \mathrm{kg} / \mathrm{dosis}$ vía intravenosa, por seis dosis.

En todo momento se atendieron complicaciones como: crisis convulsivas, hipotensión arterial (tensión arterial media por debajo de percentil 10 para edad gestacional), hipertensión pulmonar (presión pulmonar arterial por arriba de $30 \mathrm{mmHg}$ ), oliguria, coagulopatía (microembolismo, disfunción plaquetaria, incremento en la actividad fibrinolítica, prolongación de los tiempos de coagulación), infecciones u otras complicaciones.
Al término de la HT, los pacientes fueron valorados por el servicio de Neurología Pediátrica. Como parte de esta evaluación, en todos se realizó ultrasonido transfontanelar al inicio, al término y siete días después del HT. Además, entre los 10 y 14 días después de terminar la HT se realizó resonancia magnética (IRM) de cráneo. Finalmente, videoelectroencefalograma y potenciales evocados auditivos y visuales al egreso de la UCIN.

Variables de resultado. El desenlace principal fue la supervivencia; mientras que las variables secundarias fueron: evaluación neurológica, los resultados de potenciales auditivos y visuales, así como de la IRM (por ejemplo, presencia de infartos cerebrales).

\section{Análisis estadístico}

Los datos se presentan con frecuencias simples y porcentajes. Para las tasas de supervivencia se calcularon intervalos de confianza al 95\% (IC95\%). Las variables continuas fueron resumidas en medianas, con valores máximos y mínimos. Los análisis se realizaron con el paquete estadístico JASP.

Tabla 1: Características de los neonatos al nacimiento según modalidad de hipotermia terapéutica.

\begin{tabular}{|c|c|c|}
\hline & $\begin{array}{l}\text { Hipotermia } \\
\text { corporal } \\
\text { total } \\
(\mathbf{n}=\mathbf{8})\end{array}$ & $\begin{array}{l}\text { Enfriamiento } \\
\text { craneal } \\
\text { selectivo } \\
(n=4)\end{array}$ \\
\hline \multicolumn{3}{|l|}{ Sexo } \\
\hline Masculino & 5 & 4 \\
\hline Femenino & 3 & 0 \\
\hline \multicolumn{3}{|c|}{ Semanas de edad gestacional } \\
\hline \multicolumn{3}{|l|}{ Peso al nacimiento (gramos) } \\
\hline Mediana (mín-máx) & $\begin{array}{c}2,675 \\
(1,850-3,100)\end{array}$ & $\begin{array}{c}3,385 \\
(2,640-3,880)\end{array}$ \\
\hline \multicolumn{3}{|l|}{ Apgar al minuto de nacimiento } \\
\hline $0-2$ & 2 & 2 \\
\hline $3-4$ & 5 & 1 \\
\hline $5-6$ & 1 & 1 \\
\hline \multicolumn{3}{|l|}{ Apgar a los 5 minutos } \\
\hline $3-4$ & 3 & 2 \\
\hline $5-6$ & 2 & 1 \\
\hline $7-8$ & 3 & 1 \\
\hline Reanimación avanzada & 7 & 4 \\
\hline Asistencia a la ventilación & 7 & 3 \\
\hline
\end{tabular}


Tabla 2: Estudios de laboratorio al inicio de la hipotermia terapéutica, según la modalidad.

\begin{tabular}{|c|c|c|c|c|}
\hline & \multicolumn{2}{|c|}{ Hipotermia corporal total $(\mathbf{n}=8)$} & \multicolumn{2}{|c|}{ Enfriamiento craneal selectivo $(n=4)$} \\
\hline & Mediana & mín-máx & Mediana & min-máx \\
\hline pH sérico & 7.01 & $6.87-7.15$ & 6.96 & $6.99-7.15$ \\
\hline $\mathrm{pO}_{2}(\mathrm{mmHg})$ & 52.1 & $16.2-172.0$ & 78.0 & $23-225.0$ \\
\hline $\mathrm{pCO}_{2}(\mathrm{mmHg})$ & 34.0 & $18.2-86.10$ & 42.90 & $38.6-94.0$ \\
\hline $\mathrm{HCO} 3$ (mmHg) & 19.1 & $3.4-15.10$ & 11.60 & 7-17.70 \\
\hline Déficit base & -19.9 & $-26.8,-15.90$ & -17.10 & $-23.2,-13.0$ \\
\hline Lactato sérico & 12.8 & $11-20.00$ & 12.40 & $11.9-16.0$ \\
\hline$\%$ Sat $\mathrm{O}_{2}$ & 84.1 & $12.8-99.0$ & 90.80 & $26.3-99.50$ \\
\hline $\mathrm{Hb}(\mathrm{g} / \mathrm{dL})^{2}$ & 15.4 & $2.9-18.5$ & 17.7 & 16-20.1 \\
\hline $\mathrm{Na}(\mathrm{mmol} / \mathrm{L})$ & 130.5 & $120-137.0$ & 126.0 & $119-133.0$ \\
\hline Cloro (mmol/L) & 106.0 & $99-109.0$ & 103.0 & $97-111.0$ \\
\hline Glucosa (g/dL) & 57.0 & $6-148.0$ & 99.0 & $18-131.0$ \\
\hline CPK (mg/dL) & 346.0 & $138-2,970.0$ & 849.0 & $357-1,582.0$ \\
\hline CPK BB & 10.8 & 4.1-22.7 & 10.0 & 2.3-22.0 \\
\hline CPK MB & 4.6 & $0-11.1$ & 5.0 & $3-5.0$ \\
\hline Dímero D ( $\mu \mathrm{g} / \mathrm{L})$ & $5,744.0$ & $0-39,162.0$ & $1,217.0$ & $825-42,924.0$ \\
\hline Fibrinógeno (mg/dL) & 95.2 & $0-164.0$ & 117.2 & 55.5-192.3 \\
\hline
\end{tabular}

\section{Aspectos éticos}

El proyecto fue aprobado por el Comité de Investigación y Ética del hospital, con el número de registro ENS 2019 P 001.

\section{RESULTADOS}

Entre el año 2015 y 2017 ingresaron cuatro pacientes al protocolo de hipotermia con el sistema CoolCap ${ }^{\circledR}$ y entre el año 2018 y 2019 ingresaron ocho pacientes que fueron manejados con el sistema de hipotermia corporal total. Los antecedentes de los neonatos según la modalidad de hipotermia terapéutica se muestran en la Tabla 1. En ambos grupos predominaron los pacientes masculinos y los neonatos de término. Los datos de hipoxia entre los dos grupos fueron semejantes (Apgar al minuto y 5 minutos, necesidad de reanimación cardiopulmonar avanzada y el requerimiento de ventilación mecánica).

$\mathrm{El}$ inicio de la hipotermia fue en promedio a las 3 horas de vida (mín. 1:39 horas, máx. 6:00 horas). Todos los casos se encontraron en acidosis metabólica grave con hiperlactatemia (Tabla 2). En ambos grupos hubo pacientes con anemia severa, así como con hipoglucemia grave. En todos los pacientes estas alteraciones fueron corregidas antes o durante la terapia hipotér- mica. Con respecto a las enzimas $\mathrm{CPK}$ en sus diversas fracciones, en todos los pacientes se elevaron, lo mismo ocurrió con dímero D y fibrinógeno.

Las complicaciones más frecuentes durante el manejo hipotérmico (Tabla 3) fueron las alteraciones de la coagulación, hipertensión arterial pulmonar y acidosis grave persistente, las cuales fueron resueltas, por lo que no fue necesario suspender la HT. En todos, el periodo de calentamiento duró entre siete y 10 horas.

Ningún paciente falleció durante la HT; sin embargo, dos pacientes (16.6\%; IC95\%, 2.8\%-48\%) fallecieron después del procedimiento. Estos RN fueron manejados con HCT y eran de término. Uno falleció a los 13 días de vida por falla cardiaca relacionada a hipertensión arterial pulmonar, pero persistía con datos de EHI grave. El segundo falleció a los 5 días de vida, pero también tenía evidencia clínica y electroencefalografía de EHI grave; además, en IRM se observaron infartos cerebrales múltiples, con predominio en los ganglios basales.

Evaluación neurológica. Como se muestra en la Tabla 3, antes del inicio de la HT tres pacientes presentaron crisis convulsivas clínicas, las cuales se corroboraron con aEEG y cuatro más presentaron crisis durante la HT, todos ellos continuaron presentando crisis dentro de las primeras 48 horas de HT y remitieron con doble terapia anticomicial (levetiracetam y midazolam). 
Previo al inicio del enfriamiento, el ultrasonido transfontanelar fue normal en cuatro pacientes, en 7 se evidenció edema cerebral leve, y en un caso hubo presencia de hemorragia subependimaria derecha grado I. Los ultrasonidos realizados durante y al término de la HT no mostraron cambios respecto a los iniciales.

En el aEEG antes de la HT se observó patrón C (brotesupresión) en ocho pacientes (seis en HCT y uno en ECS), patrón B (discontinuo) en dos pacientes bajo ECS y patrón A (normal) en dos pacientes (uno en cada grupo).

Condiciones al egreso. De los 10 pacientes que sobrevivieron, en ocho la valoración clínica neurológica se reportó con reflejos, tono y movimientos corporales sin datos de alarma. En los dos restantes, hubo datos de alarma por alteración del tono muscular, focalización por disminución de la movilidad de alguna extremidad y reflejos alterados.

En cinco pacientes los potenciales visuales, auditivos y somatosensoriales fueron normales. En los cinco restantes se detectaron problemas, tres fueron del grupo de HCT y dos de ECS. De los tres primeros, uno presentó retraso auditivo por defecto de la conducción central, así como hipoacusia neurosensorial bilateral, otro mostró retraso auditivo por defecto de conducción tálamo cortical somático del hemisferio izquierdo, y el tercero registró defecto de conducción a nivel rostral y desincronización de las vías auditivas centrales por inmadurez en los mecanismos de mielinización. En los dos casos de ECS, uno tuvo hipoacusia neurosensorial bilateral periférica, y el segundo retraso en vía auditiva periférica izquierda.

En los 10 supervivientes se realizó IRM. En cinco, los resultados fueron reportados sin alteraciones; en tres, se observaron datos de edema, pero la exploración neurológica fue normal. Un paciente con manifestaciones clínicas de focalización motora, presentó infarto subcortical en regiones parieto-occipitales y en brazos posteriores de cápsulas internas (caso con HTC). El quinto paciente presentó infarto de tálamo caudado derecho, siendo del grupo que recibió HT mediante ECS.

\section{DISCUSIÓN}

Con los datos de este estudio se puede afirmar que, en general, la experiencia del uso de HT bajo dos modalidades en neonatos con EHI fue favorable. Por un lado, porque los procedimientos de enfriamiento fueron

Tabla 3: Evolución antes, durante y después la hipotermia terapéutica, según la modalidad.

\begin{tabular}{|c|c|c|c|}
\hline & $\begin{array}{l}\text { Hipotermia corporal total } \\
\qquad(n=8)\end{array}$ & $\begin{array}{l}\text { Enfriamiento craneal selectivo } \\
\qquad(n=4)\end{array}$ & $\begin{array}{c}\text { Ambos } \\
n(\%) \\
(n=12)\end{array}$ \\
\hline \multicolumn{4}{|l|}{ Antes hipotermia } \\
\hline Crisis convulsivas (aEEG) & 2 & 1 & $3(25)$ \\
\hline \multicolumn{4}{|l|}{ Ultrasonido transfontanelar } \\
\hline Normal & 3 & 1 & $4(33.3)$ \\
\hline Edema leve & 4 & 2 & $6(50.0)$ \\
\hline Edema severo & 0 & 1 & $1(8.3)$ \\
\hline Hemorragia intracraneal & 1 & 0 & $1(8.3)$ \\
\hline \multicolumn{4}{|l|}{ Durante la hipotermia } \\
\hline Alteración coagulación & 6 & 2 & $8(66.7)$ \\
\hline Hipertensión pulmonar & 6 & 2 & $8(66.7)$ \\
\hline Acidosis grave persistente & 5 & 2 & $7(58.3)$ \\
\hline Crisis convulsivas (aEEG) & 4 & 3 & $7(58.3)$ \\
\hline Sepsis o neumonía & 4 & 2 & $6(50.0)$ \\
\hline \multicolumn{4}{|l|}{ Evaluación después de hipotermia } \\
\hline Exploración neurológica anormal & 1 & 1 & $2(16.7)$ \\
\hline \multicolumn{4}{|l|}{ Resonancia magnética nuclear } \\
\hline Normal & 3 & 2 & $5(41.6)$ \\
\hline Edema o lesiones difusas & 2 & 1 & $3(25.0)$ \\
\hline Infartos & 2 & 1 & $3(25.0)$ \\
\hline Muerte intrahospitalaria & 2 & 0 & $2(16.7)$ \\
\hline
\end{tabular}


llevados de acuerdo con lo paneado en los 12 pacientes, sin haber defunciones durante su implementación, mientras que, por otro lado, el recalentamiento también se logró de la manera programada. Sin embargo, los pacientes desarrollaron las complicaciones esperadas, ${ }^{6,7}$ como acidosis metabólica severa, coagulopatías, trombocitopenia severa, hipertensión pulmonar, las cuales fueron corregidas.

En estudios previos ${ }^{12,13}$ no se ha observado que las modalidades de HT influyan en la supervivencia de los pacientes. En la presente investigación hubo dos pacientes que fallecieron después de ser tratados con HCT. Pero, por el número de pacientes, no es posible afirmar que esta modalidad sea mejor que ECS, dado el escaso número de participantes y diseño del estudio. Ahora bien, sin tomar en cuenta el tipo de HT, el porcentaje de supervivientes (83.4\%) de este estudio se encuentra dentro de los reportado en revisiones sistemáticas, el cual es de aproximadamente $75 \%$ (IC 95\%: 73.8\%-77.1\%). ${ }^{6,7} \mathrm{Al}$ respecto, es importante señalar que la mayor parte de los decesos suelen ocurren durante la hospitalización; sin embargo, estos pacientes pueden fallecer durante los primeros 18 meses de vida, por lo que valdría la pena continuar la vigilancia de nuestros pacientes a más largo plazo.

Con relación a las complicaciones neurológicas, la literatura disponible sugiere que la evaluación del efecto de la HT sea a los 18 meses de edad, ${ }^{6,7}$ ya que en esta edad será más preciso determinar el daño neurológico. Sin embargo, diversas publicaciones han señalado que el estudio de IRM puede ser buen predictor de secuelas neurológicas posteriores. ${ }^{14}$ Mastrangelo y colaboradores ${ }^{15}$ encontraron que en $\mathrm{RN}$ con EHI y con datos normales (o leves) en IRM tuvieron un mejor neurodesarrollo a 18 meses, a diferencia de aquéllos con lesiones moderadas o graves; en particular, si tenían anormalidades estructurales y espectroscópicas en los ganglios basales. En nuestros pacientes, un neonato con datos de daño a nivel de tálamo evidenció daño neurológico funcional en sus estudios de EEG y potenciales somatosensoriales, por lo que es posible que a largo plazo su neurodesarrollo no será normal.

En la actualidad, en países desarrollados, la HT es una recomendación vigente que debe ser usada en neonatos con EHI moderada y severa. ${ }^{11,16-18}$ A pesar de los resultados mostrados en el ámbito mundial, en Latinoamérica existen pocos reportes sobre el uso de HT; en este contexto, la presente investigación, aunque con número limitado de pacientes, constituye una de las primeras publicaciones, por lo que esperamos que contribuyan a aumentar la utilización de la HT.

\section{REFERENCIAS}

1. Lai MC, Yang SN. Perinatal hypoxic-ischemic encephalopathy. J Biomed Biotechnol. 2011; 2011: 609813. doi: 10.1155/2011/609813.

2. Manotas H. Descripción de una cohorte de pacientes neonatos con diagnóstico de asfixia perinatal, tratados con hipotermia terapéutica. Perinatol Reprod Hum. 2018; 32 (2): 70-77. doi: org/10.1016/j.rprh.2018.07.001.

3. INEC. Estadísticas Vitales Registro Estadístico de Nacidos vivos y Defunciones 2016. Disponible en: http://www. ecuadorencifras.gob.ec/documentos/web-inec/Poblacion_y_ Demografia/Nacimientos_Defunciones/2016/Presentacion_ Nacimiento s_y_Defunciones_2016.pdf.

4. Murguia SMT, Lozano R, Santos JI. Mortalidad perinatal por asfixia en México: problema prioritario de salud pública por resolver. Bol Hosp Infant Mex. 2005; 62: 375-383.

5. Moral Y, Robertson NJ. Hipoxia-isquemia neonatal: bases celulares y moleculares del daño cerebral y modulación terapéutica de la neurogénesis. Rev Neurol. 2019; 68(01): 23-36. doi: 10.33588/rn.6801.2018255.

6. Tagin MA, Woolcott CG, Vincer MJ, Whyte RK, Stinson DA. Hypothermia for neonatal hypoxic ischemic encephalopathy: an updated systematic review and meta-analysis. Arch Pediatr Adolesc Med. 2012; 166(6): 558-566. doi: 10.1001/ archpediatrics.2011.1772.

7. Jacobs SE, Berg M, Hunt R, Tarnow-Mordi WO, Inder TE, Davis PG. Cooling for newborns with hypoxic ischaemic encephalopathy. Cochrane Database of Syst Rev. 2013; 2013(1): CD003311. doi: 10.1002/14651858.CD003311. pub3.

8. McAdams RM, Juul SE. Neonatal encephalopathy: update on therapeutic hypothermia and other novel therapeutics. Clin Perinatol. 2016; 43(3): 485-500. doi: 10.1016/j. clp.2016.04.007.

9. García AA, Alarcón AA. Hipotermia terapéutica en el recién nacido a término o casi término con encefalopatía hipóxicoisquémica. An Pediatría Continuada. 2013; 11(4): 212-217. doi: 10.1016/S1696-2818(13)70140-9.

10. Nonato M, Gheler L, Balestrieri JV, Audi M, Prandini M. Selective head cooling and whole-body cooling as neuroprotective agents in severe perinatal asphyxia. Rev Assoc Med Bras. 2019; 65(8): 1116-1121. doi: 10.1590/1806-9282.65.8.1116.

11. Schump EA. Neonatal encephalopathy: current management and future trends. Crit Care Nurs Clin North Am. 2018; 30(4): 509-521. doi: 10.1016/j.cnc.2018.07.007.

12. Strohm B, Azzopardi D; UK TOBY Cooling Register Study Group. Temperature control during therapeutic moderate whole-body hypothermia for neonatal encephalopathy. Arch Dis Child Fetal Neonatal Ed. 2010; 95 (5): F373-F375. doi: 10.1136/adc.2009.163816.

13. Gulczynska EM, Gadzinowski J, Kesiak M et al. Therapeutic hypothermia in asphyxiated newborns: selective head cooling vs. whole body cooling-comparison of short-term outcomes. Ginekol Pol. 2019; 90(7): 403-410. doi: 10.5603/GP.2019.0069.

14. Sánchez-Fernández I, Morales-Quezada JL, Law S, Kim P. Prognostic value of brain magnetic resonance imaging in neonatal hypoxic-ischemic encephalopathy: a metaanalysis. J Child Neurol. 2017; 32(13): 1065-1073. doi: $10.1177 / 0883073817726681$.

15. Mastrangelo MJ, Di Marzo G, Andreoli CH, Chiara-Colajacomo M, Ruggieri A, Papoff P. Early post-cooling brain magnetic resonance for the prediction of neurodevelopmental outcome 
in newborns with hypoxic-ischemic encephalopathy. Pediatr Neurosci. 2019; 14(4): 191-202. doi: 10.4103/jpn.JPN_25_19: 10.4103/jpn.JPN_25_19.

16. Zhang W, Ma J, Danzeng Q, Tang Y, Lu M, Kang Y. Safety of moderate hypothermia for perinatal hypoxic-ischemic encephalopathy: a meta-analysis. Pediatr Neurol. 2017; 74: 51-61. doi: 10.1016/j.pediatrneurol.2017.04.023.

17. Manley BJ, Owen LS, Hooper SB et al. Towards evidencebased resuscitation of the newborn infant. Lancet.
2017; 389(10079): 1639-1648. doi: $10.1016 /$ S01406736(17)30547-0.

18. Shankaran S, McDonald SA, Laptook AR, Hintz SR, Barnes PD, Das A et al. Eunice Kennedy Shriver National Institute of Child Health and Human Development Neonatal Research Network. Neonatal magnetic resonance imaging pattern of brain injury as a biomarker of childhood outcomes following a trial of hypothermia for neonatal hypoxic-ischemic encephalopathy. J Pediatr. 2015; 167: 987-93.e3. 